\title{
Erratum
}

\section{The timing of relativistic proton acceleration in the 20 January 2005 flare, and other papers}

\author{
G. M. Simnett
}

School of Physics \& Astronomy, University of Birmingham, B15 2TT, UK

e-mail: gms@star.sr.bham.ac.uk

A\&A, 445, 715-724 (2006), DOI: 10.1051/0004-6361:20053503

A\&A, 440, 759-766 (2005), DOI: 10.1051/0004-6361:20040229

\begin{abstract}
In 2006 a timing error was discovered in the EPAM data from the ACE spacecraft, which has since been corrected. There are two publications where the interpretation of the data needs some revision on account of the error (Simnett 2006, A\&A, 445, 715; Simnett et al. 2005, A\&A, 440,759). Here we publish the correct versions of the data and indicate where the discussion in the above papers needs modifying.
\end{abstract}

Key words. acceleration of particles - Sun: coronal mass ejections (CMEs) - Sun: flares - Sun: particle emission - errata, addenda

\section{Introduction}

The EPAM instrument (Gold et al. 1998) on the Advanced Composition Explorer (ACE) spacecraft monitors low energy charged particles in the near Earth environment. Data from EPAM has been used in many publications since the launch of ACE in August, 1997. In 2006 the EPAM team was made aware of a timing drift in the EPAM data. This timing drift was $\sim 1 \mathrm{~min} / \mathrm{year}$. The team has identified the source of this drift and remedied the problem. The entire EPAM dataset has been regenerated and reference to this may be found currently on the ACE Science Center website http://www.srl.caltech.edu/ACE/ASC/news.html.

\section{Papers in Astronomy and Astrophysics which need correcting}

We are aware of two papers published in Astronomy and Astrophysics where the timing error may have led to wrong or misleading conclusions, and we now publish the necessary corrections.

\subsection{The timing of relativistic proton acceleration in the 20 January 2005 flare, G. M. Simnett, A\&A, 445, 715}

Figure 4 in this paper should be replaced by Fig. 1. In Fig. 9 the time for the pitch angle distribution in the upper panel should be changed to 06:59-07:02 UT and for the lower panel it should be 06:51-06:52 UT. In Table 1 the starting and maximum times for E4 should be 06:50 and 06:56 UT respectively, and the times at the Sun should be 06:38 and 06:44 UT respectively.

The primary conclusions of the paper are unchanged, as they refer to the high energy proton acceleration and propagation.

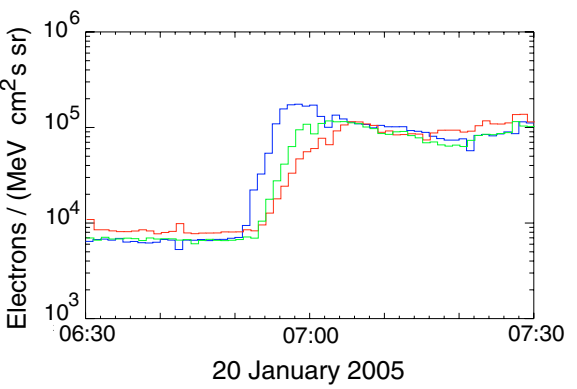

Fig. 1. The $\sim 175-\sim 300 \mathrm{keV}$ electron intensity for the peak sector in the LEFS60 (green, sector 3), LEFS150 (blue, sector 4) and the deflected electrons (red, sector 2) telescopes from 06:30-07:30 UT on 20 January. The data are plotted at a time resolution of $1 \mathrm{~min}$. (Replacement for Fig. 4 of Simnett 2006.)

We did, however, discuss the relative injection times of the high energy protons and near-relativistic electrons. With the correct EPAM timing, then within the uncertainties in the data and propagation assumptions we should conclude that the electrons and protons are injected at the Sun around the same time.

\subsection{Proton and electron acceleration by quasi-perpendicular fast magnetosonic shocks in interplanetary space, G. M. Simnett, J.-I. Sakai and R. J. Forsyth, A\&A, 440, 759}

The high time resolution EPAM/ACE data were only used in Figs. 10 and 11 of this paper. Figure 10 should be replaced by Fig. 2. The effect of the timing correction is to move the rapid onset of the shock associated electron spike to just before the arrival of the shock at ACE. The conclusions in Sect. 5 of the paper remain unchanged. 

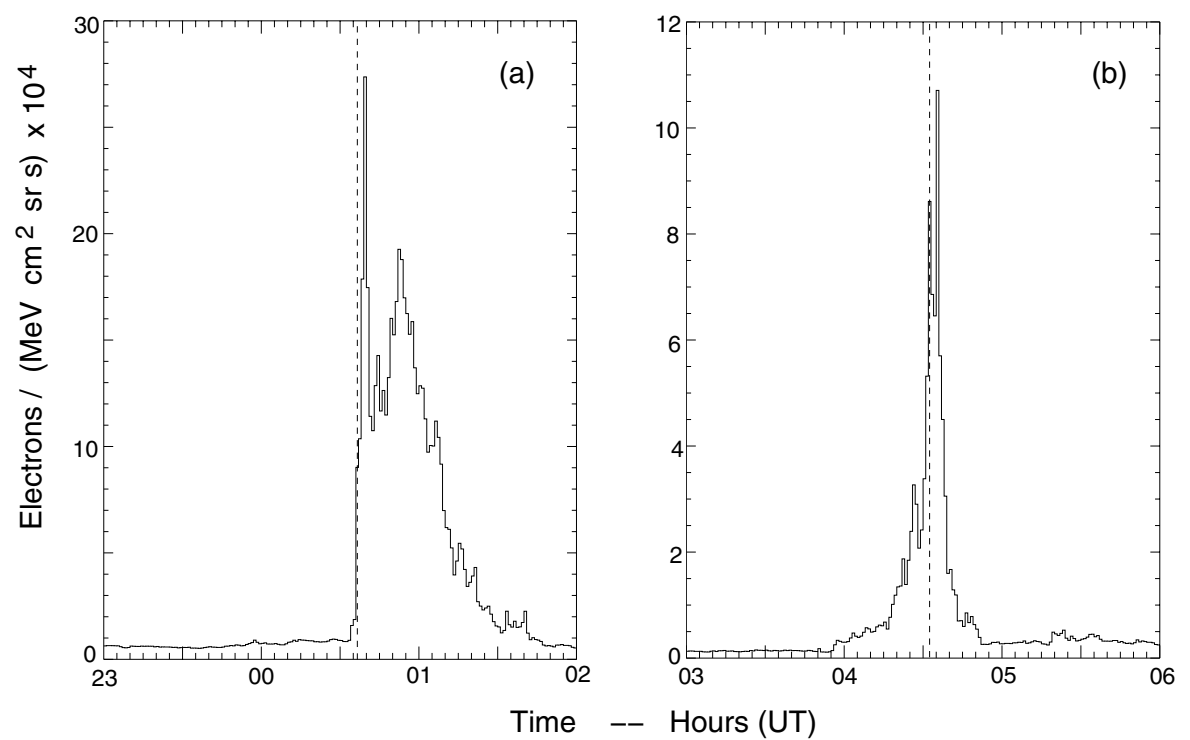

Fig. 2. The 53-103 keV electron intensity-time histories for three hours centred around the shock arrival for a) the 10 May 2001 event at Ulysses and b) the 28 April, 2001 event at ACE. The data are plotted as 1 min averages. The times of arrival at the shocks at the spacecraft are indicated by the dashed lines. (Replacement for Fig. 10 of Simnett et al. 2005.)

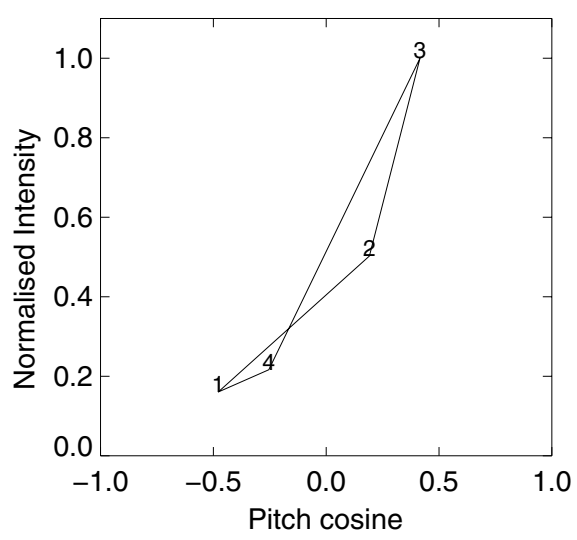

Fig. 3. The 53-103 keV electron pitch angle distribution for the $24 \mathrm{~s}$ interval of peak intensity, 04:35:30-04:35:54 UT, following the shock on 28 April, 2001. The numbers on the distribution refer to the sector of the DE2 channel plotted. (Replacement for Fig. 11 of Simnett et al. 2005.)
Figure 11 should be replaced by Fig. 3. This change does not affect the conclusions of the paper.

Acknowledgements. The author wishes to thank Dr Allan Tylka for drawing our attention to the ACE timing problem. Thanks are due to colleagues on the ACE team, and especially Drs. D. K. Haggerty and S. J. Tappin, for their help in making the corrected data available and in a useable form. Starlink computing facilities were used for the detailed analysis.

\section{References}

Gold, R. E., Krimigis, S. M., Hawkins, S. E. et al. 1998, Space Sci. Rev., 86, 541 Simnett, G. M. 2006, A\&A, 445, 715

Simnett, G. M., Sakai, J.-I., \& Forsyth, R. J. 2005, A\&A, 440, 759 